\title{
ON PERTURBATION OF UNSTABLE SECOND ORDER LINEAR DIFFERENTIAL EQUATIONS
}

\author{
L. HATVANI AND L. PINTÉR
}

\begin{abstract}
In connection with a conjecture of J. M. Bownds, conditions will be given on the fundamental system of the solutions of the unstable differential equation $y^{\prime \prime}+a(t) y=0$ which assure that the differential equation $x^{\prime \prime}+a(t) x=g\left(t, x, x^{\prime}\right)$ has a solution with the property$$
\lim \sup \left(|x(t)|+\left|x^{\prime}(t)\right|\right)=\infty \quad \text { as } t \rightarrow \infty,
$$

provided that $g\left(t, x, x^{\prime}\right)$ is "sufficiently small".
\end{abstract}

1. J. M. Bownds recently showed that from some theorems of stability theory it follows that the differential equation

$$
y^{\prime \prime}+a(t) y=0, \quad t \geqslant 0,
$$

for arbitrary $a(t) \in C[0, \infty)$ has a solution $y(t)$ with property

$$
\limsup _{t \rightarrow \infty}\left(|y(t)|+\left|y^{\prime}(t)\right|\right)>0 .
$$

He also established that the equation

$$
x^{\prime \prime}+a(t) x=g\left(t, x, x^{\prime}\right), \quad t \geqslant 0,
$$

has the above property too, provided that the zero solution $y=0$ of (1) is stable and there exists a function $\gamma(t) \in L[0, \infty)$ such that

$$
\left|g\left(t, x, x^{\prime}\right)\right| \leqslant \gamma(t)\left(|x|+\left|x^{\prime}\right|\right)
$$

for $\left(t, x, x^{\prime}\right) \in[0, \infty) \times R \times R$.

As it was remarked in [1], it seems reasonable to conjecture that the latter statement is still true if the zero solution $y=0$ of (1) is unstable.

It is well known that a necessary and sufficient condition for the zero solution of (1) to be unstable is the existence of a solution $y(t)$ with

$$
\limsup _{t \rightarrow \infty}\left(|y(t)|+\left|y^{\prime}(t)\right|\right)=\infty \text {. }
$$

In this article we prove that the conjecture of $\mathbf{J}$. M. Bownds holds under certain conditions; moreover in this case there exists a solution of (2) with property $\left(R_{2}\right)$.

2. Let us denote by $R^{2}$ the space of column vectors $z=\operatorname{col}\left(z_{1}, z_{2}\right)=$ $\left(z_{1}, z_{2}\right)^{*}$ and define the norm of $z$ by $\|z\|=\left|z_{1}\right|+\left|z_{2}\right|$. We shall denote by $y_{1}(t), y_{2}(t)$ the solutions of $(1)$ with the initial conditions $y_{1}(0)=1, y_{1}^{\prime}(0)=0$,

Received by the editors December 12, 1974 and, in revised form, September 15, 1975. AMS (MOS) subject classifications (1970). Primary 34C10, 34D10.

Key words and phrases. Perturbation, stability, instability, conditional stability. 
$y_{2}(0)=0, y_{2}^{\prime}(0)=1$ and set

$$
Y(t)=\left[\begin{array}{ll}
y_{1}(t) & y_{2}(t) \\
y_{1}^{\prime}(t) & y_{2}^{\prime}(t)
\end{array}\right]
$$

THEOREM. Suppose that the zero solution $y=0$ of (1) is unstable and there exist two projections $P_{1}, P_{2}$ on the plane and a constant $K$ such that $P_{1}+P_{2}$ $=I$ and

$$
\begin{array}{ll}
\left\|Y(t) P_{1}\left(-y_{2}(s), y_{1}(s)\right)^{*}\right\| \leqslant K, & 0 \leqslant s \leqslant t<\infty, \\
\left\|Y(t) P_{2}\left(-y_{2}(s), y_{1}(s)\right)^{*}\right\| \leqslant K, & 0 \leqslant t \leqslant s<\infty .
\end{array}
$$

Assume further that there exists a function $\gamma(t) \in L[0, \infty)$ such that

$$
\left|g\left(t, x_{1}, x_{1}^{\prime}\right)-g\left(t, x_{2}, x_{2}^{\prime}\right)\right| \leqslant \gamma(t)\left(\left|x_{1}-x_{2}\right|+\left|x_{1}^{\prime}-x_{2}^{\prime}\right|\right)
$$

for $\left(t, x_{1}, x_{1}^{\prime}\right),\left(t, x_{2}, x_{2}^{\prime}\right) \in[0, \infty) \times R \times R$.

Then equation (2) has a solution $x(t)$ with property $\left(\mathrm{R}_{2}\right)$.

Proof. Since $Y^{-1}(s)\left(0, z_{2}\right)^{*}=z_{2}\left(-y_{2}(s), y_{1}(s)\right)^{*}$, from (4) and (5) we have

$$
\begin{array}{ll}
\left\|Y(t) P_{1} Y^{-1}(s)\left(0, z_{2}\right)^{*}\right\| \leqslant K\left|z_{2}\right|, & 0 \leqslant s \leqslant t<\infty, \\
\left\|Y(t) P_{2} Y^{-1}(s)\left(0, z_{2}\right)^{*}\right\| \leqslant K\left|z_{2}\right|, & 0 \leqslant t \leqslant s<\infty
\end{array}
$$

for all $z_{2}$.

Taking into account the special form of (2), by a slight modification ${ }^{1}$ of Theorem 11 of $\left[2\right.$, p. 76] we obtain, assuming $\left(4^{\prime}\right),\left(5^{\prime}\right)$ and $\left(3^{\prime}\right)$, that there exists a one-to-one correspondence between the $\|\cdot\|$-bounded solutions $y(t)$ of (1) and $x(t)$ of (2).

Let $t_{1}$ be a sufficiently large fixed number; then this correspondence satisfies

$$
P_{1} Y^{-1}\left(t_{1}\right)\left(x\left(t_{1}\right), x^{\prime}\left(t_{1}\right)\right)^{*}=P_{1} Y^{-1}\left(t_{1}\right)\left(y\left(t_{1}\right), y^{\prime}\left(t_{1}\right)\right)^{*}
$$

and is bicontinuous in the space $C\left(\left[t_{1}, \infty\right) ; R^{2}\right)$ with the supremum norm.

First consider the case $P_{1} \neq 0$. From (4) it follows that the function $\left\|Y(t) P_{1}\right\|$ is bounded; consequently every solution $y(t)$ of (1) with the property $Y^{-1}\left(t_{1}\right)\left(y\left(t_{1}\right), y^{\prime}\left(t_{1}\right)\right)^{*} \in P_{1} R^{2}$ is $\|\cdot\|$-bounded. Moreover, from the instability of $y=0$ we obtain that all $\|\cdot\|$-bounded solutions have this property, i.e., $P_{1} \neq I$.

Therefore on the straight line $P_{1} Y^{-1}\left(t_{1}\right)\left(y, y^{\prime}\right)^{*}=P_{1}\left(c, c^{\prime}\right)^{*}\left(c\right.$ and $c^{\prime}$ are constants), there is a unique point, through which a $\|\cdot\|$-bounded solution of (1) is passing at $t=t_{1}$. Because of (6) the same is true also for equation (2). Consequently, equation (2) has a solution satisfying $\left(\mathrm{R}_{2}\right)$.

Now suppose that $P_{1}=0$, i.e., $P_{2}=I$. If every nontrivial solution of (1) is

\footnotetext{
${ }^{1}$ We modify Theorem 11 of [2] by replacing Coppel's condition (23) by

$$
\begin{array}{ll}
\left\|Y(t) P_{1} Y^{-1}(s) P\right\| \leqslant K & \text { for } t_{0} \leqslant s \leqslant t, \\
\left\|Y(t) P_{2} Y^{-1}(s) P\right\| \leqslant K & \text { for } t_{0} \leqslant t \leqslant s .
\end{array}
$$
}

Here $P$ is a projection such that $P f(i, x)=f(t, x)$. In our case if $x=\left(x_{1}, x_{1}^{\prime}\right)^{*} \in R^{2}$, then $f(t, x)=\left(0, g\left(t, x_{1}, x_{1}^{\prime}\right)\right)^{*}$. 
not $\|\cdot\|$-bounded, then from the above-mentioned theorem it follows that every solution of $(2)$ satisfies $\left(R_{2}\right)$. If $(1)$ has $\|\cdot\|$-bounded solutions, then these are passing through the points of a straight line $\mathrm{e}$ of $R^{2}$ at $t=t_{1}$ (otherwise (1) would have two linearly independent $\|\cdot\|$-bounded solutions, which contradicts the hypothesis that the solution $y=0$ is unstable) and are conditionally stable (see [2, p. 76]) with respect to the points of the straight line $\mathbf{e}$; that is, for every $\varepsilon>0$ there exists $\delta(\varepsilon)>0$ such that if $\left(y_{1}\left(t_{1}\right)\right.$, $\left.y_{1}^{\prime}\left(t_{1}\right)\right)^{*} \in \mathbf{e},\left(y_{2}\left(t_{1}\right), y_{2}^{\prime}\left(t_{1}\right)\right)^{*} \in \mathbf{e},\left|y_{1}\left(t_{1}\right)-y_{2}\left(t_{1}\right)\right|+\left|y_{1}^{\prime}\left(t_{1}\right)-y_{2}^{\prime}\left(t_{1}\right)\right|<\delta$, then $\left|y_{1}(t)-y_{2}(t)\right|+\left|y_{1}^{\prime}(t)-y_{2}^{\prime}(t)\right|<\varepsilon$ for every $t \geqslant t_{1}$. From this fact it follows that the mapping $\mathbf{e} \rightarrow C\left(\left[t_{1}, \infty\right) ; R^{2}\right)$, defined by the correspondence $\left(y\left(t_{1}\right)\right.$, $\left.y^{\prime}\left(t_{1}\right)\right)^{*} \rightarrow\left(y(t), y^{\prime}(t)\right)^{*}$, is one-to-one and continuous. On the other hand, according to the theorem cited above, the set of $\|\cdot\|$-bounded solutions $y(t)$ of (1) can be continuously and in a one-to-one manner mapped onto the set of $\|\cdot\|$-bounded solutions $x(t)$ of equation (2). Furthermore, the mapping from $C\left(\left[t_{1}, \infty\right) ; R^{2}\right.$ ) onto $R^{2}$ defined by $\left(x(t), x^{\prime}(t)\right)^{*} \rightarrow\left(x\left(t_{1}\right), x^{\prime}\left(t_{1}\right)\right)^{*}$ is obviously one-to-one and continuous. If equation (2) would not have a solution with property $\left(\mathrm{R}_{2}\right)$, then the mapping $\mathbf{e} \rightarrow R^{2}:\left(y\left(t_{1}\right), y^{\prime}\left(t_{1}\right)\right)^{*}$ $\rightarrow\left(x\left(t_{1}\right), x^{\prime}\left(t_{1}\right)\right)^{*}$ would be a one-to-one and continuous mapping of $\mathbf{e}$ onto $R^{2}$, which is impossible. Our theorem is proved.

REMARK. Conditions (4) and (5) can certainly be satisfied if $a(t)=a \neq 0(a$ is a constant), or $a(t)$ is a periodic function with period $\tau$ and $\left(y_{1}(\tau)+y_{2}(\tau)\right)^{2}$ $\neq 4$ (see $[2$, pp. 48,78$]$ ).

\section{REFERENCES}

1. J. M. Bownds, Stability implications on the asymptotic behavior of second order differential equations, Proc. Amer. Math. Soc. 39 (1973), 169-172. MR 47 \#2150.

2. W. A. Coppel, Stability and asymptotic behavior of differential equations, Heath, Boston, Mass., 1965. MR $32 \# 7875$.

Bolyai Institute, Aradi Vértanuk Tere 1, Szeged, Hungary 